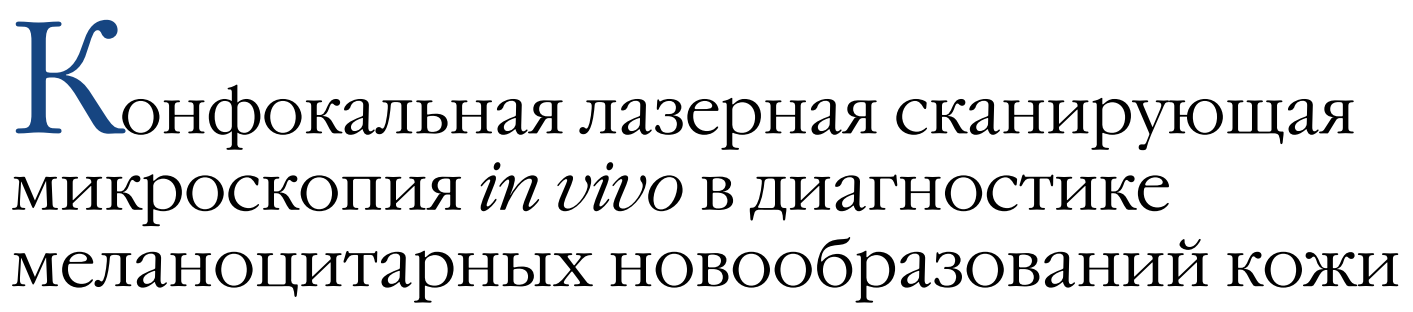

А.А. Кубанова, В.В. Чикин, Ю.Ю. Штиршнайдер, О.Р. Катунина

ФГБУ «Государственный научный центр дерматовенерологии и косметологии» Минздрава России 107076, Москва, ул. Короленко, д. 3, стр. 6

\footnotetext{
Рассмотрены возможности конфокальной лазерной сканирующей in vivo микроскопии (КЛСМ) в диагностике меланоцитарных новообразований кожи и ее значение при раннем выявлении меланомы. КЛСМ является инновационным неинвазивным методом визуального исследования кожи, позволяющим обследовать пациента в режиме реального времени, дает возможность проводить безболезненное исследование многократно, не сопровождается нарушением целостности кожных покровов. Метод позволяет с высокой чувствительностью и специфичностью обнаруживать меланому кожи на ранних этапах развития, что дает возможность использовать КЛСМ для скрининга меланоцитарных новообразований кожи и обеспечить раннее начало лечения для сохранения жизни и здоровья пациентов.
}

Ключевые слова: конфокальная лазерная сканирующая in vivo микроскопия, меланоцитарные новообразования кожи, ранняя диагностика меланомы кожи.

Контактная инфформация: chikin@mail.ru. Вестник дерматологии и венерологии 2014; (3): 85—94.

\title{
Confocal laser scanning microscopy in vivo for diagnosing melanocytic skin neoplasms
}

\author{
A.A. Kubanova, V.V. Chikin, Yu.Yu. Shtirshneider, O.R. Katunina
}

State Research Center of Dermatovenereology and Cosmetology, Ministry of healthcare of the Russian Federation Korolenko str., 3, bldg 6, Moscow, 107076, Russia

The authors discuss the use of confocal laser scanning microscopy in vivo (CLSM) for diagnosing melanocytic skin neoplasms and its value for early diagnostics of melanoma. CLSM is an innovation noninvasive visual examination method for real-time multiple and painless examinations of the patient's skin without injuring the skin integument. The method ensures early diagnostics of skin melanomas with high sensitivity and specificity, which makes it possible to use CLSM for screening melanocytic skin neoplasms for the sake of the early onset of treatment to save patient life and health.

Key words: confocal laser scanning microscopy in vivo, melanocytic skin neoplasms, early diagnostics of skin melanomas. 
Значительную долю в структуре первичной обращаемости пациентов к дерматологу составляют больные с новообразованиями на коже и слизистых оболочках. Обширную группу новообразований кожи представляют собой меланоцитарные новообразования кожи, характеризующиеся большим разнообразием нозологических фоом и их клинических проявлений. Согласно классификации меланоцитарных новообразований ВОЗ (Лион, 2006), они подразделяются на доброкачественные невусы (меланоцитарные невусы, невусы Ота, голубой невус и др.) и злокачественную меланому [1]. По данным ряда авторов, частота трансформации предшествующих меланоцитарных невусов в меланому колеблется в широких пределах - от 8,4 до 50\% случаев [2-4].

Меланома является одной из наиболее агрессивных злокачественных опухолей, которая характеризуется лимфогенным и гематогенным метастазированием и плохим прогнозом при позднем выявлении. Заболеваемость меланомой увеличивается во всем мире [5-7].

В Российской Федерации заболеваемость меланомой кожи (стандартизированные показатели) составила 3,28 на 100000 населения в 2002 г. и 3,97 на 100000 населения - в 2012 г. (рис. 1). Среднегодовой темп прироста составил 1,85\%, прирост $20,65 \%$ [8].

Хотя на долю меланомы приходится всего 10\% злокачественных новообразований кожи, большинство (80-90\%) смертей, связанных с опухолями кожи, вызвано именно меланомой [9, 10]. Смертность от меланомы кожи (стандартизированные показатели) в 2002 г. в Российской Федерации составила 1,31 на 100000 населения и 1,47 на 100000 населения в 2012 г. Анализ динамики смертности от меланомы показал, что среднегодовой темп прироста составил 1,35\%, прирост - 14,63\% (рис. 2).

Пятилетняя выживаемость больных меланомой зависит от глубины инвазии опухоли, которая может быть определена по Кларку или по Бреслоу [11].

Выделяют 5 уровней инвазии меланомы по Кларку, которые определяются при патоморфологическом исследовании:

- Уровень инвазии I: атипичные меланоциты и/или их комплексы располагаются в пределах эпидермиса (меланома in situ).

口 Уровень инвазии II: меланома пересекает эпидермис и проникает в сосочковый слой дермы (стадия микроинвазивного радиального роста).

- Уровень инвазии III: опухоль заполняет сосочковый слой дермы (начало фазы «истинной опухоли»). Такие опухоли вступают в фазу вертикального роста.

Уровень инвазии IV: опухолевые клетки проникают в сетчатый слой дермы.

- Уровень инвазии V: опухоль распространяется в подкожную клетчатку.

Согласно Бреслоу, меланому классифицируют в зависимости от ее толщины в миллиметрах:

1. Тонкая меланома (толщина опухоли составляет меньше 0,75 миллиметра).

2. Промежуточная меланома (толщина новообразования составляет 0,76-3,99 миллиметра).

3. Толстая (глубокая) меланома (толщина опухоли превышает 4 миллиметра).

Установлена четкая прямая зависимость повышения уровня смертности от толщины и глубины инвазии меланомы. Для тонких меланом с толщиной опухоли

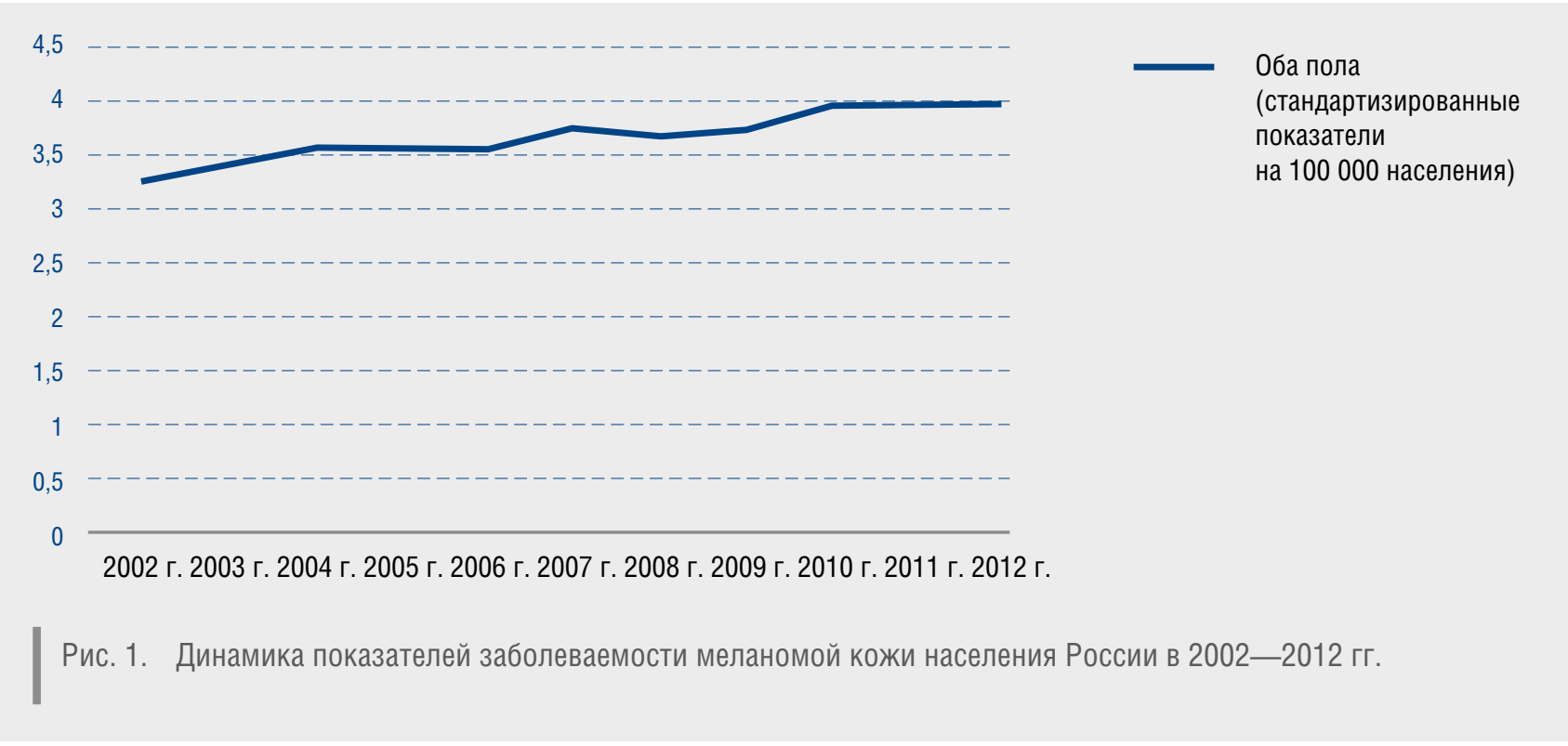




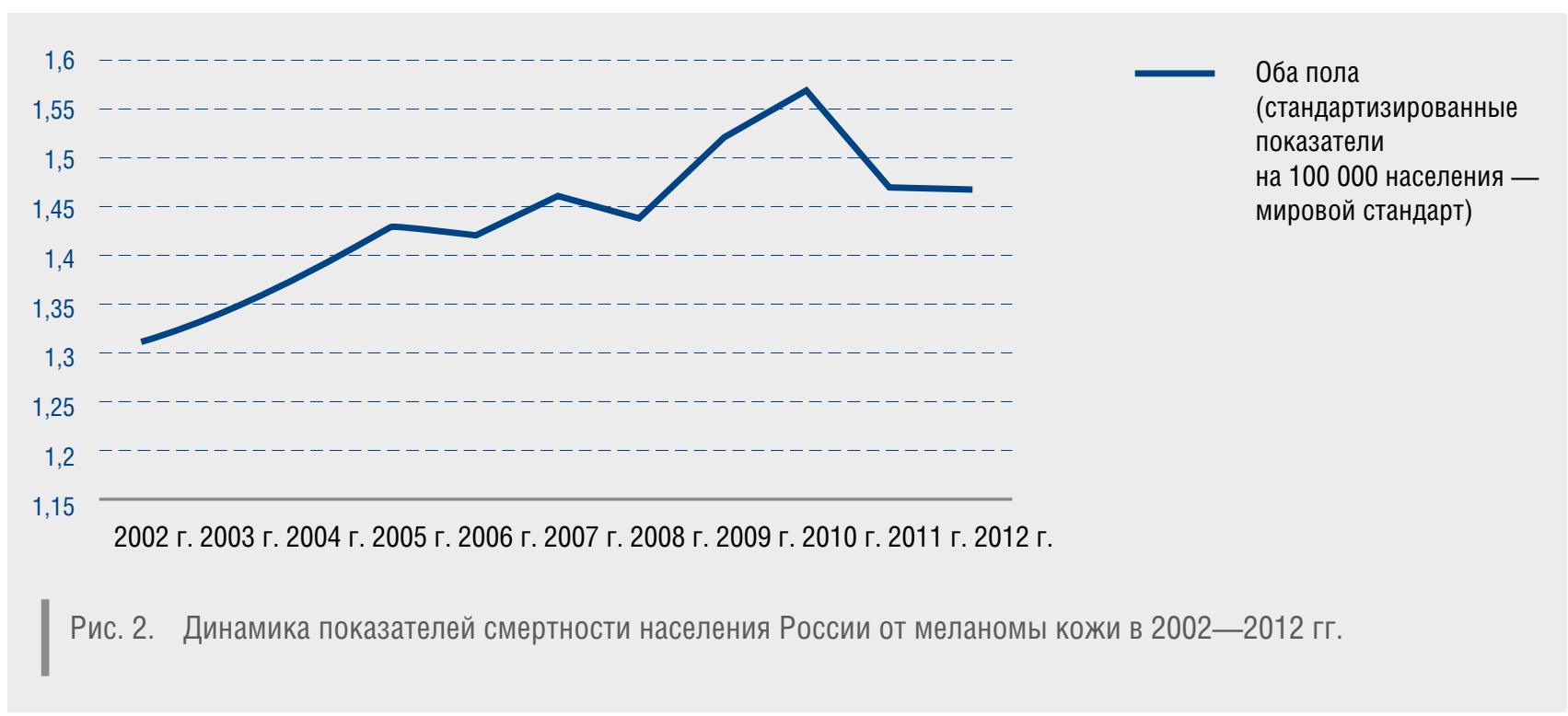

менее 0,76 мм 10-летний уровень выживаемости составляет 99,5\%. Однако этот уровень значительно уменьшается до 48\% при наличии очагов толщиной более 3 мм [12].

Неблагоприятным отличием меланом от других злокачественных новообразований кожи является возможность образования метастазов уже при небольшом объеме опухоли. Показано, что в случае образования метастаза меланомы средняя продолжительность жизни пациента составляет примерно 9 месяцев, а 5-летний уровень выживаемости составляет менее 10\% [10]. По данным P. Cinar и соавт., при наличии метастазов в лимфатические узлы 5-летняя выживаемость составляет всего 15\% [13].

Злокачественные новообразования обладают большим социальным значением, что обусловлено значительными экономическими потерями вследствие высокой стоимости лечения на поздних стадиях заболевания, инвалидизацией и преждевременной смертностью больных трудоспособного возраста.

В связи с повышением уровня смертности в случае поздней диагностики меланомы кожи большое значение приобретает ее выявление на ранних стадиях развития. Раннее выявление и своевременное иссечение злокачественного новообразования, по мнению экспертов Меланомной Программы ВОЗ, лежат в основе успеха терапии больного и являются залогом обеспечения хорошего прогноза выживаемости и снижения смертности [14]. Своевременная диагностика опухолей позволяет сократить число запущенных случаев, снизить риск глубокой инвазии и рецидива опухоли, тем самым улучшая качество жизни пациентов после проведенного лечения.

Хотя новообразования кожи относятся к опухолям, доступным для визуализации, их диагностика сложна как для начинающих онкологов, не имеющих достаточного опыта в интерпретации поражений кожи, так и для дерматологов, которые не всегда проявляют достаточную онкологическую настороженность [15]. Сложность диагностики опухолей кожи обусловлена не только многообразием нозологических форм, но и тем, что одна и та же опухоль может иметь ряд клинических разновидностей. Указывая на огромное разнообразие новообразований кожи, с которым сталкивается практический врач, М.Л. Гельфонд делает вывод об отсутствии сколько-нибудь стройной и логичной системы практических мероприятий, способной в полной мере удовлетворять требованиям как хирургов, дерматологов, косметологов, с одной стороны, так и онкологов - с другой, что приводит к различной, а подчас и прямо противоположной лечебной тактике онкологов и косметологов в отношении опухолей кожи [15]. В первом случае даже безобидное новообразование рассматривается как потенциально злокачественное и удаляется в кратчайшие сроки, с соблюдением всех принципов хирургической онкологии с морфологической верификацией удаленной опухоли [18]. Во втором - существующее у пациента новообразование кожи рассматривается прежде всего как косметический дефект, и объем лечебных мероприятий диктуется соображениями максимальной выгоды с косметической, а нередко - коммерческой точки зрения [19].

Диагностика меланоцитарных новообразований кожи основывается на данных клинического обследования пациента и подтверждается гистологическим исследованием.

Одним из наиболее известных симптомокомплексов, используемых в диагностике меланомы, является клиническое правило ABCD [20]. Правило ABCD вклю- 
чает визуальную оценку пигментного новообразования кожи по 4 критериям:

А - асимметрия пигментного пятна;

В - неровность границ;

C - неравномерность окраски;

D - диаметр более 6 мм.

Наличие трех и более признаков говорит о злокачественности новообразования. В 1999 г. дополнительно был введен критерий $\mathrm{E}$ (evolution) для оценки изменений цвета, формы и размера пигментного образования кожи при динамическом наблюдении за пациентом.

Тем не менее используемое при клиническом обследовании правило ABCD характеризуется низкой специфичностью, так как другие, в том числе доброкачественные, новообразования кожи, например себорейный кератоз, могут полностью отвечать этим критериям. D. Rigel и соавт. (2010) указывают, что чувствительность клинической диагностики меланомы с помощью правила ABCD варьирует от 57 до 90\%, а специфичность - от 59 до 90\% [21].

Клиническое правило ABCD не позволяет диагностировать меланому кожи на ранней стадии развития, когда толщина опухоли по Бреслоу не превышает 1 мм, а также небольшие меланомы диаметром 6 мм и менее. Кроме того, правило ABCD неприменимо для пигментных образований, располагающихся на ладонях, подошвах и лице из-за особенностей анатомии кожи в этих участках. Беспигментные и узловые формы меланомы также могут быть исключением из этого правила.

Л.В. Демидов и соавт. указывают, что в России точность клинической диагностики врачами первичного звена при первичном обращении составляет $30 \%$ [23].

Для подтверждения диагноза злокачественного новообразования необходимо проведение гистологического исследования. Приказом Минздрава России от 20 декабря 2012 года № 1143Н утвержден стандарт первичной медико-санитарной помощи при злокачественных новообразованиях кожи (меланома, рак) I-IV стадии (обследование в целях установления диагноза и подготовки к противоопухолевому лечению), в соответствии с которым для уточнения диагноза «меланома» предусмотрено обязательное проведение гистологического исследования препарата кожи, поскольку оно позволяет детально охарактеризовать клеточные структуры, образующие опухоль, определить ее гистогенез, доброкачественность или злокачественность процесса, уточнить степень инвазии [24]. Но осуществить гистологическое исследование можно только после иссечения новообразования. Дерматологи, особенно работающие в учреждениях частной фрормы собственности, которые не всегда имеют в распоряжении патоморфологическую лабораторию, проводят удаление новообразований в амбулаторных условиях методом тканевой деструкции, так как не всегда владеют навыками хирургического иссечения.

Для гистологического исследования характерна также отсроченность получения результатов, особенно в лечебных учреждениях, не обладающих собственной патоморфологической лабораторией. Ятрогенная травма при диагностической, лечебно-диагностической тотальной биопсии с последующим формированием рубцовой деформации кожи не всегда желательна в случаях локализации процесса на лице. Кроме того, при подозрении на меланому не рекомендуется инцизионная биопсия кожи.

В этой связи одним из важных стратегических подходов к своевременному выявлению меланоцитарных новообразований кожи является их ранняя диагностика путем проведения профилактических и скрининговых мероприятий. Для этого применяют неинвазивные методы исследования кожных покровов, которые позволяют получить информацию об изучаемом объекте - кожных покровах и слизистых оболочках без нарушения его целостности.

K существующим на сегодняшний день методам неинвазивной диагностики новообразований кожи относятся:

дерматоскопия;

высокочастотное ультразвуковое сканирование;

конфокальная лазерная сканирующая in vivo микроскопия.

Дерматоскопия - метод, успешно используемый врачами разных специальностей, основан на принципе исследования пигментного образования на поверхности кожи in vivo при среднем увеличении объекта в 10—40 раз с использованием эффекта эпилюминисценции.

Изучены специфические дерматоскопические признаки доброкачественных невусов и меланомы кожи, касающиеся изменения цвета, архитектоники, формы, симметрии, границ, которые положены в основу диагностических алгоритмов, позволяющих проводить диагностику $[25,26]$. А. Gerger и соавт. приводят данные, что чувствительность дерматоскопии в диагностике меланомы кожи по сравнению с визуальной диагностикой выше на 10-27\% [27].

Дерматоскопия позволяет изучить структуру кожных покровов, включая сосочковый слой дермы, но ограничивает возможности оценки новообразования в случае его залегания в более глубоких слоях кожи и в подкожно-жировой клетчатке. Кроме того, дерматоскопия не позволяет различать патологические изменения в клеточных структурах, являющиеся одним из важных признаков для диагностики новообразований кожи.

Высокочастотное ультразвуковое сканирование кожи основано на взаимодействии ультразвука и тканей человека, которые обладают различными акустическими характеристиками. Получение изобра- 
жения происходит благодаря излучению ультразвуковых импульсов, направленных в исследуемые ткани, и обработке отраженных сигналов. Для ультразвукового исследования кожных покровов используются специальные датчики, частота которых (20-100 МГц) отличается от частоты датчиков, используемых для ультразвукового исследования внутренних органов (до 20 МГц). Метод позволяет визуализировать структуры кожи до глубины 8-10 мм, включая все еe слои: эпидермис, дерму, подкожно-жировую клетчатку, определять количественные параметры: толщину эпидермиса и дермы, размеры сканируемых образований, их границы и глубину залегания, акустическую плотность различных структур и патологических очагов [28-31]. В то же время использование этой технологии не позволяет отличить меланому кожи от меланоцитарного невуса из-за схожести эхографических признаков доброкачественных и злокачественных новообразований.

Конфокальная лазерная сканирующая in vivo микроскопия (КЛСМ) - это принципиально новое направление в изучении структур кожи, занимающее особое место среди неинвазивных визуализирующих технологий. Метод основан на компьютерном анализе различий коэфрфициента отражения (рефракции) света для различных структур кожи.

Для КЛСМ используется лазерный луч, направленный вглубь ткани, с одной (830 нм (ближняя инфракрасная область)) или несколькими (785 нм (ближняя инфракрасная область), 658 нм (красная область), 488 нм (синяя область)) длинами волн и мощностью не более 40 мВт. При использовании конфокального лазерного микроскопа сканирование кожи начинается с эпидермиса и происходит послойно. Глубина визуализации новообразования зависит от длины волны лазерного излучения. При длине волны лазера 488 нанометров она достигает 50 нм. При длине волны 1064 нанометра - $400 \mu \mathrm{M}$, что позволяет визуализировать эпидермис, сосочковый и сетчатый слои дермы.

Метод позволяет неинвазивно, быстро, в режиме реального времени получить контрастные изображения «оптических» срезов эпидермиса и верхней части дермы на клеточном уровне, ориентированных в горизонтальной плоскости с разрешением, сопоставимым с обычной световой микроскопией [32-39]. Получение горизонтальных «оптических» срезов в плоскости, параллельной поверхности кожи, а не вертикальных, характерных для гистологического исследования, является отличительной особенностью изображения, полученного с помощью конфокальной лазерной сканирующей микроскопии.

Изображение, полученное посредством конфокального микроскопа, представляет собой двухмерную картину бело-серо-черных оттенков. Структуры, содержащие меланин и кератин (меланоциты, кератиноциты), интенсивнее всего отражают свет, поэтому на изображении выглядят ярко-белыми [40, 41]. Воздух, серозная жидкость, не отражающие свет, окрашены в черный цвет. Диагностика меланоцитарных новообразований методом конфокальной лазерной сканирующей in vivo микроскопии основана на свойстве меланина интенсивно отражать свет. Меланин является лучшим эндогенным контрастным веществом в организме человека.

Четкость конфокально-микроскопического изображения достигается путем отсекания апертурой микроскопа фонового света, идущего из глубины образца, то есть того света, который не попадает в фокальную плоскость объектива микроскопа, и попадания в детектор прибора только того света, который находится в фокусной плоскости. Отсюда и название метода «конфокальный», или «софрокусный». В результате изображение получается более контрастным, чем в обычном оптическом микроскопе. Неинвазивность технологии позволяет проводить многократное наблюдение пациента в динамике.

Благодаря тому что КЛСМ обладает разрешением, приближенным к традиционной световой микроскопии, с помощью этого метода можно различать клетки разных слоев эпидермиса, сосочкового и сетчатого слоев дермы, оценивать состояние капилляров дермы и придатков кожи [40-49]. Метод позволяет оценить размеры клеток, глубину их расположения, толщину слоев эпидермиса, состояние отдельных волокон сосочкового слоя и верхней части сетчатого слоя дермы, диаметр просветов капилляров, а также размеры отдельных клеток крови в просветах капилляров [37, 40-42]. С помощью КЛСМ можно идентифицировать меланоциты, пигментированные кератиноциты, меланофаги. Типичные параметры конфокальной микроскопии в сравнении с гистологическим исследованием кожи представлены в таблице [50].

Показано, что с помощью метода КЛСМ можно различать доброкачественные меланоцитарные невусы и меланому кожи [25, 47, 51-53].

Установлено, что в доброкачественных меланоцитарных невусах архитектоника слоев эпидермиса остается сохранной. Края кератиноцитов определяются четко. Ярко-белые меланоциты всегда мономорфны, имеют округлые или овальные очертания. Сосочки дермы имеют четкие границы, в которых наблюдаются гнездные скопления меланоцитов, одинаковых по размеру и фрорме (рис. 3, 4) [46, 52, 54].

При КЛСМ диспластических меланоцитарных невусов наряду с признаками доброкачественности могут обнаруживаться признаки атипии: определяются ярко-белые меланоциты крупных размеров, различной формы, границы гнездных скоплений меланоцитов (кластеров) выражены менее отчетливо, местами не определяются границы кератиноцитов.

При меланоме кожи в изображениях, полученных при КЛСМ, часто отмечаются такие изменения, как 
Таблица Сравнение основных параметров конфокальной микроскопии in vivo и гистологического исследования [50]

\begin{tabular}{|c|c|c|}
\hline Параметр & Конфрокальная микроскопия & Гистологическое исследование \\
\hline Длина волны & $\begin{array}{l}\text { Избирательная, } \\
\text { от } 400 \text { до } 1064 \text { нм }\end{array}$ & $\begin{array}{c}\text { Широкополосный } \\
\text { белый свет, 400-700 нм }\end{array}$ \\
\hline Максимальная глубина изображения & 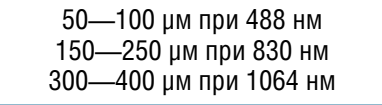 & \\
\hline Толщина среза & $\begin{array}{c}1-5 \mu \text {, } \\
\text { Неинвазивный, оптический }\end{array}$ & $\begin{array}{c}5 \mu \mathrm{m}, \\
\text { физический }\end{array}$ \\
\hline Латеральное разрешение & $0,1-1 \mu \mathrm{m}$ & $0,1-4 \mu \mathrm{M}$ \\
\hline Размер апертуры & $0,7-1,4$ & $0,1-1,4$ \\
\hline Иммерсионная среда & Вода или масло & Воздух или масло \\
\hline Увеличение & $40-100 x$ & $1-1000 x$ \\
\hline Исследовательское поле & $0,5-0,2 \mathrm{~mm}$ & $20-0,2$ мм \\
\hline Контрастирующий механизм & $\begin{array}{c}\text { Отражение света эндогенными } \\
\text { микроструктурами }\end{array}$ & Абсорбция экзогенных красителей \\
\hline Контрастный агент & $\begin{array}{c}\text { Меланин } \\
\text { Кератин } \\
\text { Коллаген }\end{array}$ & $\begin{array}{l}\text { Гематоксилин и эозин } \\
\text { Метиленовый синий } \\
\text { Толуидиновый синий }\end{array}$ \\
\hline
\end{tabular}

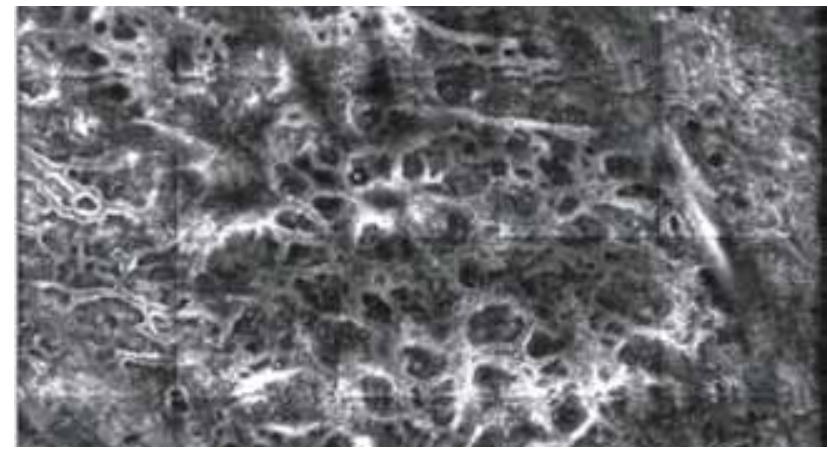

Рис. 3. Меланоцитарный невус. Конфокальномикроскопическое изображение in vivo. Дермальные сосочки в виде четких колец, одинаковых по форме и размеру

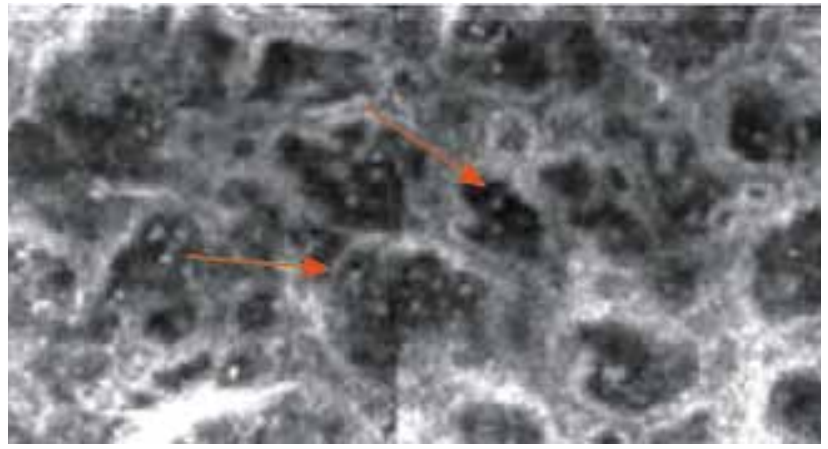

Меланоцитарный невус. Конфокальномикроскопическое изображение in vivo. Клетки внутри просветов сосочков одинаковой формы и размера (стрелка) выраженное нарушение архитектоники эпидермиса. Конфокально-микроскопическая картина шиповатого слоя эпидермиса, в норме представленная в виде пчелиных сот или «булыжной мостовой», часто нарушается за счет нечетких границ и изменения размеров кератиноцитов, появления меланоцитов с признаками атипии. При меланоме на уровне базального и шиповатого слоев эпидермиса обнаруживаются отдельно лежащие меланоциты или их гнездные скопления (рис. 5), имеющие крупные дендритные отростки при- чудливого вида, или представляющие собой крупные ярко-белые округлой формы образования. Меланин в очаге поражения распределен неравномерно (рис. 6). Признаком инвазивного роста меланомы являются яркие округлые, овальные или дендритные структуры, соответствующие атипичным меланоцитам, которые могут быть обнаружены в зоне дермо-эпидермального соединения и в верхних слоях дермы (педжетоидное распространение). Границы дермальных сосочков могут быть нечеткими (рис. 7), и визуализируемые в их 

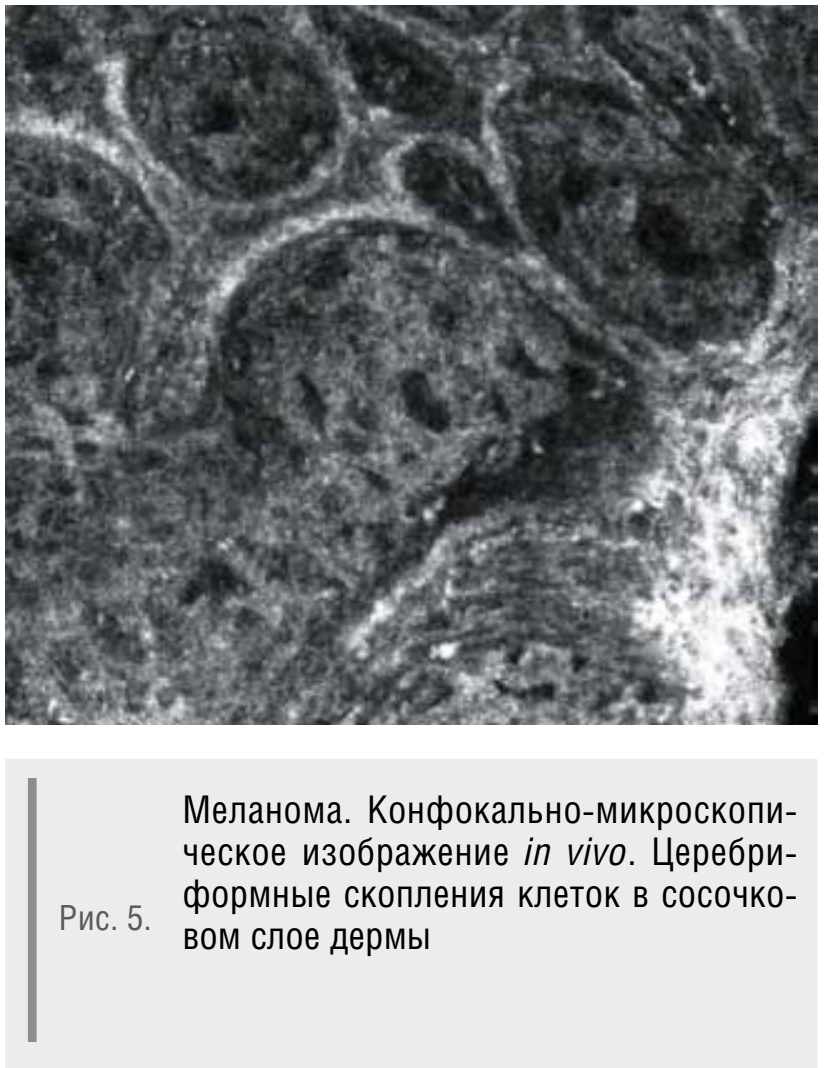

просвете меланоциты могут содержать ядра (рис. 6) $[26,51]$. Об усилении ангиоматоза может свидетельствовать изменение ориентации сосудов, расположенных в горизонтальной плоскости относительно поверхности кожи, в которых увеличивается скорость кровотока (рис. 7).

Показано, что чувствительность КЛСМ в диагностике меланомы кожи составляет 88,15\%, а специфичность - 97,6\%. В исследовании 117 меланоцитарных новообразований кожи с использованием трех диагностических критериев исследователи правильно идентифицировали меланому в 96,3\% случаев, а доброкачественные невусы - в 98,89\% случаев [52]. Метаанализ, проведенный А. Stevenson и соавт., показал, что чувствительность КЛСМ в диагностике меланомы кожи составляет 93\%, а специфичность - 76\% [53].

Некоторые специфические признаки могут быть определены и в беспигментных меланомах. Меланоциты в клинически беспигментных меланомах могут обнаруживаться с помощью КЛСМ за счет того, что остаточный меланин обеспечивает достаточное контрастирование цитоплазмы [54]. Но в связи с тем, что исследования проводились на небольших по величине выборах, в настоящий момент не представляется возможным оценить чувствительность и специфичность диагностики беспигментной меланомы методом КЛСМ.

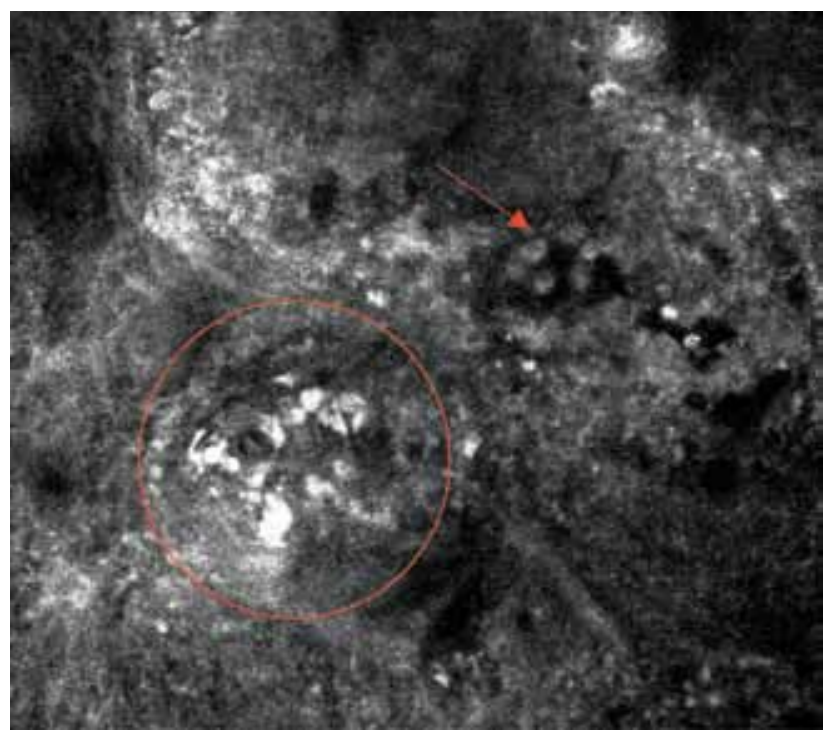

Меланома. Конфокально-микроскопическое изображение in vivo. Меланоци-

Рис. 6. ты, содержащие ядра, внутри дермальных сосочков (стрелка). Клетки различны по форме и размеру. Распределение пигмента неравномерно (круг)

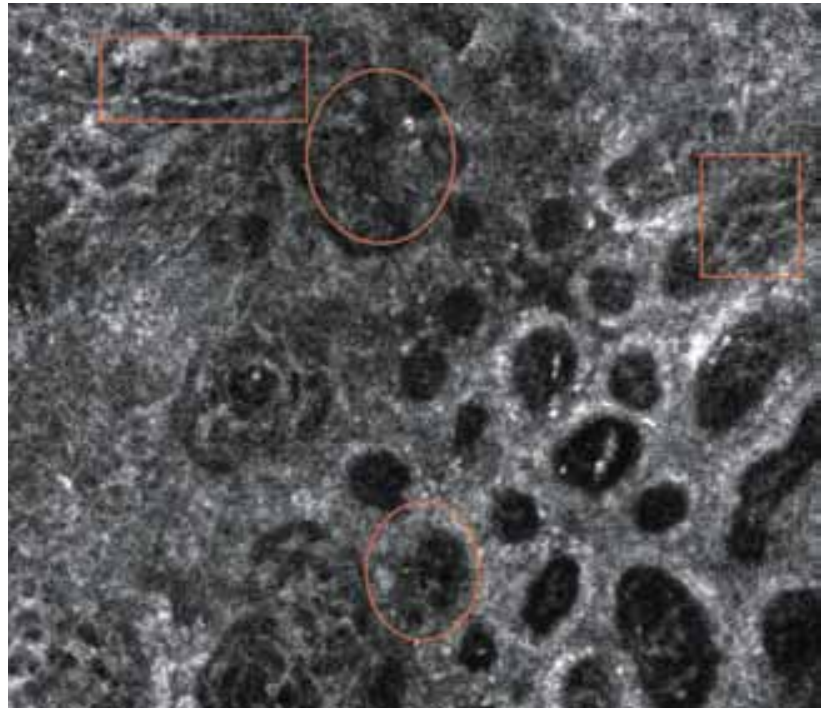

Меланома. Конфокально-микроскопическое изображение in vivo. Сосочки дермы в области эпидермально-дермальной

Рис. 7. границы без четких границ (круг). Сосуды, располагающиеся в горизонтальной плоскости по отношению к поверхности кожи (ангиоматоз) (прямоугольник) 
Обнаруженные с помощью конфокального микроскопа отличительные признаки, характерные для меланоцитарных невусов и меланомы кожи, были сопоставлены с результатами гистологического исследования, что выявило высокую корреляцию между результатами, полученными при использовании этих методов в диагностике меланоцитарных новообразований кожи $[55,56]$.

\section{Заключение}

Возможности конфокального микроскопа in vivo в диффреренциальной диагностике меланоцитарных новообразований кожи демонстрируют, что конфокальная лазерная сканирующая микроскопия является уникальным высокочувствительным и специфичным методом исследования, позволяющим диагностировать меланому кожи без нарушения целостности кожных покровов и определить дальнейшую тактику ведения пациента в самые короткие сроки после установления диагноза [15]. Кроме ранней диагностики меланоцитарных новообразований кожи возможности КЛСМ позволяют многократно обследовать пациентов после удаления опухоли с целью подтверждения радикальности проведенного лечения и выявления ее рецидива [57].

Конфокальный микроскоп in vivo позволяет в режиме реального времени фиксировать, оценивать и анализировать изменения в структуре и функции кожи, а также наблюдать за развитием заболевания и динамикой лечения. Основным преимуществом конфокальной микроскопии по сравнению с традиционным гистоологическим исследованием является сохранение целостности кожных покровов во время диагностических процедур [58], в связи с чем процедура безболезненна и не приводит к образованию рубцов. Это делает КЛСМ незаменимой в диагностике новообразований, располагающихся на лице и других областях, где проведение биопсии может быть затруд- нительным по эстетическим соображениям и по соображениям хирургической доступности. Метод характеризуется быстрым получением результата, тогда как полученный для гистологического исследования биоптат кожи должен пройти ряд этапов обработки перед тем, как он будет изучен с помощью световой микроскопии. Кроме того, в отличие от гистологического исследования КЛСМ не приводит к изменению тканей и образованию артефактов.

Возможности метода в диагностике меланоцитарных новообразований кожи ограничены рядом его технических особенностей. Отчетливые изображения структур кожи могут быть получены только до определенной глубины, в зависимости от используемой в приборе длины волны лазерного излучения, после чего качество изображения значительно снижается, делая их бесполезными. Это ограничивает его использование в случаях, когда поражение кожи распространяется на глубокие слои дермы. Частыми причинами получения некачественных изображений является присутствие косметики в области очага поражения, воздушные пузырьки, чрезмерные движения пациента и системы крепления к коже конфокального микроскопа [59]. Иногда отмечают, что проведение процедуры КЛСМ требует много времени - до 10 минут, чтобы получить изображение, и дополнительно - время, чтобы его интерпретировать [30].

Таким образом, использование КЛСМ в обследовании больных с меланоцитарными новообразованиями кожи дает возможность быстро, не прибегая к оперативному вмешательству, установить диагноз и определить необходимость использования в последующем инвазивных методов диагностики и лечения. Способность КЛСМ установить диагноз меланомы на ранних этапах ее развития позволяет использовать этот метод для скрининга меланоцитарных новообразований кожи, что может сохранить жизнь и здоровье пациентов после своевременно проведенного лечения. I

\section{Литература}

1. LeBoit P.E., Burg G., Weedon D, Sarasain A. (Eds.): World Health Organization Classification of Tumours. Pathology and Genetics of Skin Tumours. IARC Press: Lyon 2006.

2. Tannous Z.S., Mihm M.C. Jr, Sober A.J., Duncan L.M. Congenital melanocytic nevi: Clinical and histopathologic features, risk of melanoma, and clinical management. J Am Acad Dermatol 2005; 52: 197-203.

3. Togawa Y., Nakamura Y., Kamada N. et al. Melanoma in association with acquired melanocytic nevus in Japan: a review of cases in the literature. Int J Dermatol 2010; 49: 1362—1367.
4. Fernandes N.C. The risk of cutaneous melanoma in melanocytic nevi. An Bras Dermatol 2013; 88 (2): $314-315$.

5. Chen S.T., Geller A.C., Tsao H. Update on the epidemiology of melanoma. Curr Dermatol Rep 2013; 2 (1): 24-34.

6. Wong J.R., Harris J.K., Rodriguez-Galindo C., Johnson K.J. Incidence of childhood and adolescent melanoma in the United States: 19732009. Pediatrics 2013; 131 (5): 846-854.

7. Leiter U., Garbe C. Epidemiology of melanoma and nonmelanoma skin cancer the role of sunlight. Adv Exp Med Biol 2008; 624: 89_103.
8. Zlokachestvennye novoobrazovaniya v Rossii v 2012 godu (zabolevaemost' i smertnost') / Pod red. A.D. Kaprina, V.V. Starinskogo, G.V. Petrovoy. M: FGBU «MNIOI im. P.A. Gertsena» Minzdrava Rossii; 2014, 250 s. [Злокачественные новообразования в России в 2012 году (заболеваемость и смертность) / Под ред. А.Д. Каприна, В.В. Старинского, Г.В. Петровой. М: ФГБУ «МНИОИ им. П.А. Герцена» Минздрава России; 2014, 250 с.] 
9. Gilyazutdinov I.A., Khasanov R.Sh., Safin I.R., Moiseev N.V. Zlokachestvennye opukholi myagkikh tkaney i melanoma kozhi. M: Prakticheskaya meditsina, 2010; 204. [Гилязутдинов И.А., Хасанов Р.Ш., Сафин И.Р., Моисеев Н.В. Злокачественные опухоли мягких тканей и меланома кожи. М: Практическая медицина 2010; 204.]

10. Brehmer F., Ulrich M., Haenssle H.A. Strategies for early recognition of cutaneous melanoma - present and future. Dermatol Pract Concept 2012; 2 (3): 29-37.

11. Gordeladze A.S., Novitskaya T.A. Melanotsitarnye opukholi. Chast' 1. Melanotsitarnye opukholi kozhi (rabochie standarty patologoanatomicheskogo issledovaniya) / Pod red. prof. G.B. Koval'skogo // Biblioteka patologoanatoma. Nauch.-praktich. zhurnal. SPb.: GUZ «GPAB», 2009. Vyp. 111. - 48 s. [Горделадзе А.С., Новицкая Т.А. Меланоцитарные опухоли. Часть 1. Меланоцитарные опухоли кожи (рабочие стандарты патологоанатомического исследования) / Под ред. профр. Г.Б. Ковальского // Библиотека патологоанатома. Науч.практич. журнал. СПб: ГУЗ «ГПАБ», 2009. Вып. 111.48 с.]

12. Friedman R.J., Gutkowicz-Krusin D., Farber M.J. et al. The diagnostic performance of expert dermoscopists vs a computer-vision system on small-diameter melanomas. Arch Dermatol 2008; 144: 476-482.

13. Cinar P., Zell J.A., Taylor T.H. et al. Pediatric and AYA invasive cutaneous melanoma: populationbased study comparing adult melanoma cases. $\mathrm{J}$ Clin Oncol (ASCO Meeting Abstracts) 2008; 26: 9071.

14. Avril M.F., Cascinelli N., Cristofolini M. et al. Clinical diagnosis of Melanoma:-W.H.O. Melanoma Programme Publications: russkaya versiya / Pod red. L.V. Demidova, 1994, 28 s. [Клиническая диагностика меланомы кожи. Меланомная программа B03. Avril M.F., Cascinelli N., Cristofolini M. et al.: русская версия / Под ред. Л.В. Демидова, 1994, 28 с.]

15. Gel'fond M.L. Differentsial'naya diagnostika opukholey kozhi v praktike dermatologov i kosmetologov. [Practical Oncology 2012; 13 (2): 69-79. Гельфонд М.Л. Дифференциальная диагностика опухолей кожи в практике дерматологов и косметологов. Практическая онкология 2012; 13 (2): 69_79.]

16. Dubenskiy V.V., Red'ko R.V., Garmonov A.A. Novoobrazovaniya kozhi v praktike dermatovenerologa / Pod red. V.V.Dubenskogo. Tver': 000 «Triada», 2002; 148 s. [Дубенский В.В., Редько Р.В., Гармонов А.А. Новообразования кожи в практике дерматовенеролога / Под ред. В.В. Дубенского. Тверь: 000 «Триада», 2002; 148 C.]

17. Rager E.L., Bridgeford E.P., Ollila D.W. Cutaneous melanoma: update on prevention, screening, diagnosis, and treatment. Am Fam Phys 2005; 72 (2): 269-276.
18. Anisimov V.V., Gel'fond M.L., Barchuk A.S. Znachenie khirurgicheskogo i lazernogo udaleniya dobrokachestvennykh novoobrazovaniy kozhi v ambulatornoy praktike. Ambulatornaya khirurgiya 2001; 1: 24-29. [Анисимов В.В., Гельфронд М.Л., Барчук А.С. Значение хирургического и лазерного удаления доброкачественных новообразований кожи в амбулаторной практике. Амбулаторная хирургия 2001; (1): 24-29].

19. MellettD.R. Khirurgiya po Mokhsu // Sekrety dermatologii / Pod red. D.E.Fittspatrika, D.L.Elinga: per. s angl. M.-SPb: «Binom» - «Nevskiy Dialekt», 1999; 397-400. [Меллетт Д.Р. Хирургия по Мохсу // Секреты дерматологии / Под ред. Д.Е.Фитцпатрика, Д.Л.Элинга: пер. с англ. М.-СПб: «Бином» — «Невский Диалект», 1999; 397-400.]

20. Friedman R., Rigel D., Kopf A. Early detection of malignant melanoma: role of physician examination and self-examination of the skin. CA Cancer J Clin 1985; 35: 130-151.

21. Rigel D.S., Russak J., Friedman R. The evolution of melanoma diagnosis: 25 years beyond the ABCDs. CA Cancer J Clin 2010; 60: 301—316.

22. Semiletova Yu.V., Anisimov V.V., Vagner R.I. Lechenie bol'nykh pervichnoy melanomoy kozhi. Sovremennoe sostoyanie problemy. [Семилетова Ю.В., Анисимов В.В., Вагнер Р.И. Лечение больных первичной меланомой кожи. Современное состояние проблемы. Сибирский онкологический журнал 2010; 4: 41—47.]

23. Demidov L.V., Sokolov D.V., Bulycheva I.V. et al. The progress in the diagnosis of cutaneous melanoma. Journal of N.N. Blokhin Russian Cancer Research Center RAMS 2007; 18 (1): 36-41. [Демидов Л.В., Соколов Д.В., Булычева И.В. и др. Совершенствование методов диагностики меланомы кожи. Вестник РОНЦ им. Н.Н. Блохина РАМН 2007; 18 (1): 36-41.]

24. Prikaz Minzdrava Rossii ot 20.12.2012 N 1143n "Ob utverzhdenii standarta pervichnoy medikosanitarnoy pomoshchi pri zlokachestvennykh novoobrazovaniyakh kozhi (melanoma, rak) I-IV stadii (obsledovanie v tselyakh ustanovleniya diagnoza zabolevaniya i podgotovki k protivoopukholevomu lecheniyu)" (Zaregistrirovano v Minyuste Rossii 05.03.2013 № 27454). [Приказ Минздрава России от 20.12.2012 № 1143н «0б утверждении стандарта первичной медико-санитарной помощи при злокачественных новообразованиях кожи (меланома, рак) I-IV стадии (обследование в целях установления диагноза заболевания и подготовки к противоопухолевому лечению)» (Зарегистрирован в Минюсте России 05.03.2013 № 27454).]

25. Menzies S.W., Ingvar C., Crotty K. et al. Frequency and morphologic characteristics of invasive melanomas lacking specific surface microscopic features. Arch Dermatol 1996; 132 : 1178-1182.
26. Argenziano G., Fabbrocini G., Carli P. et al. Epiluminescence microscopy for the diagnosis of doubtful melanocytic skin lesions. Comparison of the $A B C D$ rule of dermatoscopy and a new sevenpoint checklist based on pattern analysis. Arch. Dermatol 1998; 134: 1563-1570.

27. Gerger A., Koller S., Kern T. et al. Diagnostic applicability of in vivo confocal laser scanning microscopy in melanocytic skin tumors. J Invest Dermatol 2005; 124: 493-498.

28. Gropper C.A., Stiller M.J., Shupak J.L. et al. Diagnostic high-resolution ultrasound in dermatology. Int J Dermatol 1993; 32: 234-250.

29. Harland C.C., Bamber J.C., Gusterson B.A. et al. High frequency, high resolution B-scan ultrasound in the assessment of skin tumours. $\mathrm{Br} \mathrm{J}$ Dermatol 1993; 128: 525—532.

30. Wassef C., Rao B.K. Uses of non-invasive imaging in the diagnosis of skin cancer: an overview of the currently available modalities. Int J Dermatol 2013; 52: 1481-1489.

31. Kardynal A., Olszewska M. Modern non-invasive diagnostic techniques in the detection of early cutaneous melanoma. J Dermatol Case Rep 2014; 1: 1-8.

32. Ferris L.K., Harris R.J. New diagnostic aides for melanoma. Dermatol Clin 2012; 30 (3): $535-545$.

33. Abramovits W., Stevenson L.C. Changing paradigms in dermatology: new ways to examine the skin using noninvasive imaging methods. Clin Dermatol 2003; 21: 353-358.

34. Rajadhyaksha M., González S., Zavislan J. et al. In vivo confocal scanning laser microscopy of human skin II: advances in instrumentation and comparison to histology. J Invest Dermatol 1999; 113: 293-303.

35. González S. Confocal reflectance microscopy in dermatology: promise and reality of non-invasive diagnosis and monitoring. Actas Dermosifiliogr 2009; 100 (Suppl. 2): 59—69.

36. Longo C., Zalaudek I., Argenziano G., Pellacani G. New directions in dermatopatology: in vivo confocal microscopy in clinical practice. Dermatol Clin 2012; 30 (4): 799_814.

37. Corcuff P., Bertrand C., Leveque J.L. Morphometry of human epidermis in vivo by real-time confocal microscopy. Arch Dermatol Res 1993; 285: 475-481.

38. Ulrich M., Lange-Asschenfeldt S. In vivo confocal microscopy in dermatology: from research to clinical application. J Biomed Opt 2013; 18 (6): 061212.

39. Tavoloni Bragaa J.C., Caob T., Olivierob M.C. et al. In vivo confocal microscopy: a promising diagnostic method for cutaneous oncology. J Clin Exp Dermatol Res 2012; S3: 002.

40. Rajadhyaksha M., Grossman M., Esterowitz D. et al. In vivo confocal scanning laser microscopy of human skin: melanin provides strong contrast. J Invest Dermatol 1995; 104: 946—952.

41. Gonzalez S., Swindells K., Rajadhyaksha M., Torres A. Changing paradigms in dermatology: confocal microscopy in clinical and surgical dermatology. Clin Derm 2003; 21: 359—369. 
42. Branzan A.L., Landthaler M., Szeimies R.-M. In vivo confocal scanning laser microscopy in dermatology. Lasers Med Sci 2007; 22: 73—82.

43. Rudnicka L., Olszewska M., Rakowska A. In vivo reflectance confocal microscopy: usefulness for diagnosing hair diseases. J Dermatol Case Report 2008; 4: 55-59.

44. Rajadhyaksha M., Anderson R.R.,Webb R.H. Video-rate confocal scanning laser microscope for imaging human tissues in vivo. Appl Opt 1999; 38: 1-12.

45. Huzaira M., Rius F., Rajadhyaksha M. et al. Topographic variations in normal skin, as viewed by in vivo reflectance confocal microscopy. J Invest Dermatol 2001; 116: 846_ 852.

46. Busam K.J., Charles C., Lee G., Halpern A.C. Morphologic features of melanocytes, pigmented keratinocytes and melanophages by in vivo confocal scanning laser microscopy. Mod Pathol 2001; 14 (9): 862_868.

47. Sauermann K., Clemann S., Jaspers S. et al. Age related changes of human skin investigated with histometric measurements by confocal laser scanning microscopy in vivo. Skin Res Technol 2002; 8: 52-56.

48. Sauermann K., Jaspers S., Koop U., Wenck H. Topically applied vitamin $\mathrm{C}$ increases the density of dermal papillae in aged human skin. BMC Dermatol 2004; 4 (1): 13.
49. Yamashita T., Kuwahara T., Gonzalez S., Takahashi M. Non-invasive visualization of melanin and melanocytes by reflectance-mode confocal microscopy. J Invest Dermatol 2005; 124 : 235-240.

50. Reflectance confocal microscopy of cutaneous tumors. Ed. by S. Gonzalez, M. Gill, A.C. Halpern, Informa Healhcare, 2008, 275 P

51. Pellacani G., Cesinaro A.M., Seidenari S. Reflectance-mode confocal microscopy of pigmented skin lesions-improvement in melanoma diagnostic specificity. J Am Acad Dermatol 2005; 53: $979-985$

52. Langley R.G., Rajadhyaksha M., Dwyer P.J. et al. Confocal scanning laser microscopy of benign and malignant melanocytic skin lesions in vivo. J Am Acad Dermatol 2001; 45: 365—376.

53. Stevenson A.D., Mickan S., Mallett S., Ayya M. Systematic review of diagnostic accuracy of reflectance confocal microscopy for melanoma diagnosis in patients with clinically equivocal skin lesions. Dermatol Pract Concept 2013; 3 (4): $19-27$.

54. Busam K.J., Hester K., Charles C. et al. Detection of clinically amelanotic malignant melanoma and assessment of its margins by in vivo confocal scanning laser microscopy. Arch Dermatol 2001; 137: 923—929.
55. Pellacani G., Longo C., Malvehy J. et al. In vivo confocal microscopic and histopathologic correlations of dermoscopic features in 202 melanocytic lesions. Arch Dermatol 2008; 144 (12): 1597-1608.

56. Pellacani G., Longo C., Ferrara G. et al. Spitz nevi: in vivo confocal microscopic features, dermatoscopic aspects, histopathologic correlates and diagnostic significance. J Am Acad Dermatol 2009; 60: 236-247.

57. Gangley R., Walsh N., Sutherland A.E. et al. The diagnostic accuracy of in vivo confocal scanning laser microscopy compared to dermascopy of benign and malignant melanocytic lesions: a prospective study. Dermatology 2007; 215: 365-372.

58. Shtirschneider Y.Y., Michenko A.V., Katunina O.R., Zubarev A.R. Up-to-date non-invasive visualization technologies in dermatology. Vestn Dermatol Venerol 2011; 6: 41—53. [Штиршнайдер Ю.Ю., Миченко А.В., Катунина О.Р., Зубарев А.Р. Современные неинвазивные технологии визуализации в дерматологии. Вестник дерматологии и венерологии 2011; (6): 41-53].

59. Curchin C.E., Wurm E.M., Lambie D.L. et al. First experiences using reflectance confocal microscopy on equivocal skin lesions in Queensland. Australas J Dermatol 2011; 52: 89—97.

об авторах:
А.А. Кубанова - д.м.Н., профессор, академик РАН, директор ФГБУ «ГНЦДК» Минздрава России, Москва
В.В. Чикин — к.м.Н., старший научный сотрудник отдела дерматологии ФГБУ «ГНЦДК» Минздрава России, Москва
Ю.Ю. Штиршнайдер — к.м.н., врач ультразвуковой диагностики консультативно-диагностического центра ФГБУ «ГНЦДК» Минздрава России, Москва
О.Р. Катунина - д.м.Н., зав. патоморфологической лабораторией ФГБУ «ГНЦДК» Минздрава России, Москва

\section{Конфликт интересов}

Авторы заявляют об отсутствии потенциального конфлликта интересов, требующего раскрытия в данной статье 